\title{
Competencias del ingeniero industrial en la Industria 4.0
}

\section{Competences of Industrial Engineers in Industry 4.0}

Isidro Jesús González- Hernández (*) https://orcid.org/0000-0003-2805-6674

Rafael Granillo-Macías (*) https://orcid.org/0000-0002-1015-667X

(*) Universidad de Quintana Roo

(Recibido: 28 de octubre de 2018; Aceptado para su publicación: 4 de marzo de 2019)

Cómo citar: González-Hernández, I. J. y Granillo-Macías, R. (2020). Competencias del ingeniero industrial en la Industria 4.0. Revista Electrónica de Investigación Educativa, 22, e30, 1-14. https://doi.org/10.24320/redie.2020.22.e30.2750

\section{Resumen}

El contexto actual de la Industria 4.0 está cambiando la formación de los ingenieros, de tal forma que las universidades implementan estrategias y acciones para adaptar los planes de estudio a los requisitos de la nueva revolución industrial. En este trabajo se realizó una investigación cualitativa de carácter bibliográfico y documental para identificar las principales competencias que debe desarrollar un ingeniero industrial, posteriormente se realizó un análisis curricular tomando como ejemplo a una universidad mexicana incluida en el Ranking Mundial de Universidades 1001+. Los resultados muestran que se deben desarrollar competencias específicas basadas en las Tecnologías de la Información y la Comunicación, como big data, Internet de la cosas y Simulación, con el propósito de interactuar en los ambientes inteligentes que propone la Industria 4.0.

Palabras clave: Industria 4.0, Ingeniería industrial, Ingeniería de la producción, Competencias.

\section{Abstract}

The current context of Industry 4.0 is changing engineering education, with universities implementing strategies and actions to adapt curricula to the needs of the new industrial revolution. This study consisted in conducting qualitative bibliographic and documentary research to identify the main competences industrial engineers need to develop, followed by an analysis of the curriculum of a Mexican university ranked 1001+ in the World University Rankings. The results show a need to develop specific competences based on information and communication technologies like big data, the Internet of Things, and simulation, in order to interact in the smart environments proposed by Industry 4.0.

Keywords: Industry 4.0, Industrial engineering, Competencies.

\section{Introducción}

Debido al rápido desarrollo de la digitalización y la robótica, los sistemas de fabricación global se están moviendo hacia la cuarta revolución industrial, conocida como Industria 4.0, es decir, la industria se está volviendo inteligente, conectada e integrada a lo largo de la cadena de suministro (Liboni et al., 2019). El concepto de Industria 4.0 tiene como objetivo crear fábricas inteligentes donde se implementan e integran tecnologías de fabricación como sistemas cibernéticos, el Internet de las cosas (IOT, por sus siglas en inglés), Cómputo en la nube, Grandes datos o big data, Analítica, entre otras. De tal forma que la nueva era tecnológica está transformando las cadenas de valor de la industria, las cadenas de valor de la producción 
y los modelos de negocios (Zhong et al., 2017).

El uso y la aplicación de estas tecnologías emergentes implican en algunos casos un alto nivel de complejidad. Por lo que se requiere de un cierto nivel de competencias en la formación universitaria para enfrentar los desafíos de los nuevos sistemas de fabricación que está generando la Industria 4.0 (Motyl et al., 2017).

Desde un enfoque multidisciplinario, un ingeniero industrial realiza una diversidad de tareas y funciones en la empresa. Mesquita et al. (2015) mencionan que las áreas de formación específicas para un ingeniero industrial comprenden funciones de la administración de la producción (incluyendo el diseño de sistemas de producción), la automatización, aseguramiento y control de calidad, ingeniería económica, investigación de operaciones, sistemas de información y cómputo, factores humanos y ergonómicos, logística, mantenimiento, administración de proyectos, sustentabilidad, diseño de productos y simulación.

Cada una de estas áreas de formación conlleva al desarrollo de competencias, consideradas como herramientas de competitividad que se promueven, principalmente, en las universidades. Bajo este contexto, uno de los proyectos más relevantes en educación superior en latinoamérica es el "Proyecto Tuning-América Latina 2004-2007", cuyo objetivo es desarrollar perfiles profesionales en términos de competencias genéricas y específicas. Este proyecto define el término competencia como el conjunto de conocimientos, habilidades y destrezas, tanto genéricas como específicas, que debe reunir un titulado para satisfacer plenamente las exigencias de los contextos sociales (Tuning-América Latina, 2007). Por otra parte, Sánchez et al. (2018) mencionan que el término competencia se refiere a la capacidad comprobada de usar el conocimiento y las habilidades personales, sociales y metodológicas en el desarrollo profesional y personal. Por lo tanto, la competencia puede entenderse como la combinación de conocimiento y experiencia.

Las competencias genéricas imponen habilidades y prácticas transversales apropiadas para la mayoría de las áreas de formación y se pueden aplicar a una variedad de trabajos, situaciones o tareas. Las competencias específicas garantizan la adquisición de conocimientos en cada área de estudio. Por ejemplo, realizar la planeación y programación de la producción de una empresa es una competencia de dominio específico del ingeniero industrial, mientras que la capacidad de trabajar en equipo es una competencia genérica (Tuning-América Latina, 2007).

En este sentido, las competencias genéricas y específicas que se desarrollan en las universidades son clave en los graduados, ya que les permite tener la capacidad de establecerse y afianzarse en campos profesionales en constante cambio (Zlatkin-Troitschanskaia et al., 2017).

Con base en la tendencia de la Industria 4.0, en este trabajo se presenta una investigación documental con el objetivo de identificar las competencias que requiere desarrollar un ingeniero industrial basadas en las Tecnologías de la Información y la Comunicación (TIC) con el propósito de interactuar en los ambientes inteligentes que propone la Industria 4.0.

\section{Método}

La metodología de este trabajo incluye una investigación documental basada inicialmente en una revisión de la literatura sobre el contexto y las competencias que se requiere desarrollar para la ingeniería industrial en el entorno de la Industria 4.0. Luego, con el propósito de determinar las competencias de la Industria 4.0 que ya están siendo abordadas por los planes de estudio en ingeniería industrial, explícita o implícitamente, se realizó un análisis curricular tomando como ejemplo a una de las principales universidades mexicanas, incluida en el Ranking Mundial de Universidades 1001+, de acuerdo al ranking International (Times Higher Education, 2018).

\section{Contexto de la Industria 4.0}

La Industria 4.0 es un nuevo capítulo en el desarrollo humano, esta revolución está habilitada por los 
avances y la fusión de tecnologías, así como por una creciente armonización e integración entre las disciplinas de investigación, en donde los avances en investigación aprovechan la capacidad tecnológica y digital. Además, la Industria 4.0 está fusionando los mundos físico, digital y biológico (Foro Económico Mundial, 2019).

El término Industria 4.0 fue acuñado en 2011 por una iniciativa del gobierno alemán con universidades y empresas privadas. Esta iniciativa fue concebida como un programa estratégico para desarrollar sistemas de producción avanzados con el objetivo de aumentar la productividad y la eficiencia de la industria. De tal forma que el concepto representa una nueva etapa en los sistemas de fabricación y producción al integrar un conjunto de tecnologías emergentes y convergentes que agregan valor a todo el ciclo de vida del producto (Frank et al., 2019).

Bajo este contexto, el término Industria 4.0 está arraigado en el concepto de manufactura inteligente, donde los sistemas de fabricación y producción se extienden desde la fabricación inteligente hasta la logística inteligente, esto incluye la digitalización de la fabricación, los dispositivos conectados, la cadena de suministro colaborativa, la toma de decisiones integrada, los sensores avanzados y el análisis de datos (Ramingwong y Manopiniwes, 2019).

De acuerdo con el Foro Económico Mundial (2019), las principales tecnologías impulsoras de la Industria 4.0 son: inteligencia artificial y robótica, neurociencia, tecnologías de la información, cadena de bloques, economía digital y sociedad, manufactura aditiva, realidad virtual y aumentada, sistemas de fabricación y producción, materiales avanzados, y biotecnología. Otro aspecto importante de esta industria es la integración vertical y horizontal.

La integración vertical se refiere a la integración de sistemas avanzados de TIC en los diferentes niveles jerárquicos de una organización, es decir, integra sistemas de producción cibernéticos desde el proveedor hasta el cliente y desde la administración hasta el taller para lograr una cadena de valor totalmente automatizada. Por otro lado, la integración horizontal consiste en la colaboración entre empresas, con intercambio de recursos e información en tiempo real (Chong et al., 2018; Dalenogare et al., 2018).

Desde la perspectiva de los planes de gobierno se han creado iniciativas y estrategias para desarrollar e impulsar la Industria 4.0 en el mundo, por ejemplo: en Alemania se aprobó el plan de acción Estrategia de alta tecnología 2020 (High-Tech Strategy 2020), así como la iniciativa Plataforma Industria 4.0 (Plattform Industrie 4.0); Estados Unidos cuenta con la estrategia Asociación de Fabricación Avanzada 2.0 (Advanced Manufacturing Partnership 2.0); Francia tiene el programa La nueva Francia industrial (La Nouvelle France Industrielle); Reino Unido presentó una imagen a largo plazo para su sector manufacturero hasta el 2050, denominado El futuro de la manufactura (Future of Manufacturing); China emitió la estrategia Hecho en China 2025 (Made in China 2025) junto con el plan Internet Plus; Japón adoptó el 5o. Plan Básico de Ciencia y Tecnología (5th Science and Technology Basic Plan), donde pone especial atención al concepto de "Sociedad Súper Inteligente" (Super Smart Society).

Según el Foro Económico Mundial (2018b), 25 países están bien posicionados para beneficiarse de la Industria 4.0, ya que están a la vanguardia en diseño, pruebas, y son pioneros de las tecnologías emergentes de la Industria 4.0: Alemania, Austria, Bélgica, Canadá, China, Dinamarca, Eslovenia, España, Estados unidos, Estonia, Finlandia, Francia, Irlanda, Israel, Italia, Japón, Malasia, Países Bajos, Polonia, República Checa, República de Corea, Singapur, Suecia, Suiza y Reino Unido.

Por lo tanto, los avances tecnológicos que fundamentan la Industria 4.0, requieren que los estudiantes de ingeniería desarrollen competencias genéricas y específicas para adaptarse a los nuevos sistemas de manufactura inteligente que está impulsado la cuarta revolución industrial.

\subsection{Competencias en la Industria 4.0}

Como resultado de un mayor uso de las tecnologías digitales en la Industria 4.0, el entorno de los empleos estará cambiando los perfiles de trabajo y, por lo tanto, requiere que los egresados de ingeniería estén 
equipados con una amplia gama de competencias. El Foro Económico Mundial (2018a) menciona que las diez habilidades o competencias genéricas necesarias para prosperar en la Industria 4.0 son: solución de problemas complejos, pensamiento crítico, creatividad, administración de personas, coordinación con otros, inteligencia emocional, juicio y toma de decisiones, orientación de servicio, negociación y flexibilidad cognitiva.

Por otra parte, Grzybowska y Lupicka (2018) realizan una revisión de la literatura con el propósito de encontrar una respuesta a la pregunta: ¿Qué tipo de competencias necesitan los gerentes e ingenieros contemporáneos para enfrentar los nuevos desafíos en la Industria 4.0?, en esta revisión los autores identificaron ocho competencias genéricas: creatividad, pensamiento emprendedor, resolución de problemas, resolución de conflictos, toma de decisiones, capacidad de análisis, habilidades de investigación y orientación a la eficiencia.

Terrés et al. (2017) mencionan que las competencias genéricas que destacan para la Industria 4.0 son: creatividad, aprendizaje, comunicación, trabajo en equipo, liderazgo, toma de decisiones, adaptabilidad y resiliencia personal. De igual forma, Acosta (2016) menciona que para el caso de los profesionales de las áreas de ingeniería, además de los conocimientos científicos y tecnológicos, se requieren competencias para trabajar en grupos multidisciplinarios y en entornos multilingües, así como capacidad para analizar y valorar el impacto social y medioambiental de las soluciones técnicas. Por su parte, Gronau et al. (2017) mencionan que las habilidades interpersonales más relevantes para esta nueva revolución industrial son las competencias de organización, procesos (de negocio, de fabricación), e interacción.

Con respecto a las competencias específicas, el Foro Económico Mundial (2018b) menciona que éstas deberán estar enfocadas en las tecnologías emergentes como: inteligencia artificial y robótica, internet de las cosas, realidad virtual y aumentada, manufactura aditiva, cadena de bloques y tecnología de registros distribuidos, materiales avanzados y nanomateriales, captura-almacenamiento y transmisión de energía, nuevas tecnologías informáticas, biotecnologías, geoingeniería, neurotecnología y tecnologías espaciales.

Por ejemplo, Ghobakhloo (2018) menciona que desde la perspectiva de la administración de recursos humanos, el primer paso para el éxito a la transición de la Industria 4.0 es la evaluación de las competencias, de tal forma que las empresas deben evaluar cuidadosamente las habilidades de su fuerza laboral y reconocer las habilidades digitales entre los empleados, e identificar las habilidades de las que carece la compañía.

Prifti et al. (2017) presentan un análisis a través de una revisión de la literatura sobre el empleo de competencias para empleados con educación superior en la Industria 4.0, el análisis está orientado a tres enfoques del conocimiento: sistemas de información, tecnología de la información e ingeniería. Los autores identificaron 64 competencias para el perfil del profesional en la Industria 4.0; sin embargo, mencionan que no se puede esperar que una sola persona posea todas las competencias mencionadas.

En su estudio, Terrés et al. (2017) aplicaron una encuesta a 22 empresas líderes en la utilización de soluciones tecnologías con el fin de identificar las competencias específicas que impactan en la Industria 4.0. Las competencias más destacadas en los niveles de supervisión, según los autores, son las relacionadas con la interacción con interfaces modernas, el conocimiento de las organizaciones y la capacidad de análisis de datos.

Para el caso de los profesionales del área de ingeniería, Jerman et al. (2018) y Terrés et al. (2017) mencionan que las principales competencias con respecto a experiencia y uso de tecnología en la Industria 4.0 son: desarrollo de sistemas, integración de tecnologías heterogéneas, tecnologías móviles, sensores/sistemas embebidos, tecnología de red/Comunicación M2M (Máquina a Máquina), robótica/inteligencia artificial, ciencias computacionales y mantenimiento predictivo.

Con base en algunos trabajos publicados sobre la Industria 4.0, en las tablas I y II se presentan las principales competencias genéricas y específicas, respectivamente, requeridas para enfrentar los desafíos de la industria 4.0 en el contexto de los sistemas de manufactura inteligentes. 
Tabla I. Competencias genéricas para la Industria 4.0

\begin{tabular}{|c|c|c|c|c|c|c|c|c|c|c|c|}
\hline & Comp & tenc & & & & & & & & & \\
\hline Autores & SPC & PC & IYC & AP & TEI & $\mathrm{IE}$ & ATD & $\mathrm{HL}$ & AYO & РYOT & $\mathrm{HI}$ \\
\hline Foro Económico Mundial (2018a) & - & - & - & - & - & - & - & - & - & - & - \\
\hline Liboni et al. (2019) & - & & & - & - & - & & - & & & \\
\hline Prifti et al. (2017) & - & & - & ! & ! & & - & - & - & - & - \\
\hline Grzybowska y Lupicka (2018) & - & & - & & & & - & & & & - \\
\hline Dalenogare et al. (2018) & - & & & & & & - & - & & - & \\
\hline Gronau et al. (2017) & & & & - & & & & - & - & - & - \\
\hline Mourtzis (2018) & - & & - & . & - & & - & & - & - & - \\
\hline Jerman et al. (2018) & - & & & ! & - & & - & & - & - & \\
\hline Terrés et al. (2017) & - & & - & & - & & - & & & - & \\
\hline
\end{tabular}

Tabla II. Competencias específicas para la Industria 4.0

\begin{tabular}{|c|c|c|c|c|c|c|c|c|c|c|c|c|}
\hline \multirow[b]{2}{*}{ Autores } & \multicolumn{12}{|c|}{ Competencias } \\
\hline & IAR & IC & RVA & MA & $\mathrm{CB}$ & BDAD & $\mathrm{s}$ & $\mathrm{IHV}$ & SC & LN & SCF & M2M \\
\hline Foro Económico Mundial (2018b) & - & - & - & - & ! & - & - & - & ! & ! & ! & - \\
\hline Frank et al. (2019) & & - & & & & - & & & & - & & \\
\hline Zhong et al. (2017) & - & - & - & & & - & & & & - & - & \\
\hline Prifti et al. (2017) & - & - & - & - & & - & - & - & - & - & . & - \\
\hline Saucedo-Martínez et al. (2017) & - & ! & - & - & & - & - & - & ! & - & & \\
\hline Dalenogare et al. (2018) & - & - & & - & & - & - & & & - & & \\
\hline Ghobakhloo (2018) & - & - & - & - & & - & - & & ! & ! & . & \\
\hline Sauza et al. (2017) & - & - & & & & - & - & & & . & & \\
\hline Onar et al. (2018) & - & & & - & & - & & & & ! & - & \\
\hline Jerman et al. (2018) & - & - & - & & & - & & & - & & - & \\
\hline Terrés et al. (2017) & घ & & & घ & & & - & - & & ! & घ & घ \\
\hline
\end{tabular}

Notas: Inteligencia Artificial y Robótica (IAR); Internet de las Cosas (IC); Realidad Virtual y Aumentada (RVA); Manufactura Aditiva (MA); Cadena de Bloques (CB); Big data y Análisis de Datos (BDAD); Simulación (S); Integración Horizontal y Vertical del sistema (IHV); Seguridad Cibernética (SC); La nube (LN); Sistemas Ciber-Físicos (SCF); Comunicación M2M (M2M).

\subsection{Competencias del ingeniero industrial en la Industria $\mathbf{4 . 0}$}

El ingeniero industrial puede diagnosticar problemas empresariales y adoptar métodos científicos para resolver el problema; así como aumentar la eficiencia de la producción, mejorar la calidad del producto, reducir el costo del producto y aumentar los beneficios para la empresa. Es decir, la ingeniería industrial es una disciplina relacionada con la tecnología y la administración.

Debido a que la Industria 4.0 tiene como elemento central la manufactura inteligente, las operaciones de fabricación harán uso de la ciencia y de las tecnologías emergentes con el objetivo de mejorar en gran medida el diseño, la producción, la administración y la integración de todo el ciclo de vida de un producto. Por lo tanto, la manufactura inteligente representa un reto para el ingeniero industrial, ya que requiere de ciertas competencias genéricas y específicas para enfrentar el desafío de la Industria 4.0. 
En este sentido, Sackey et al. (2017) realizaron un estudio para examinar los impactos de la Industria 4.0 en la formación y el currículo de ingenieros industriales con el objetivo de determinar las mejoras curriculares en los programas de estudio para adaptarse a los requisitos de la Industria 4.0 en las universidades de Sudáfrica. A partir de una revisión bibliográfica y un formulario de recolección de datos curriculares de los programas de estudio de ingeniería industrial, los autores argumentan que las siguientes áreas necesitan ser incluidas en los planes de estudio de la carrera: ciencia de datos y analítica avanzada (big data), simulación avanzada y modelado de plantas virtuales, comunicación de datos y redes, automatización de sistemas, nuevas interfaces hombre-máquina, manufactura aditiva, sistemas de control/administración de calidad de procesos y productos integrados de circuito cerrado, inventario en tiempo real y sistemas de optimización logística.

Por otra parte, Karre et al. (2017) identificaron las competencias específicas que debe desarrollar un ingeniero industrial: las habilidades y conocimientos en TIC, procesamiento y análisis de datos, conocimiento estadístico, entendimiento organizacional, habilidades para interactuar con interfaces modernas, conocimiento administrativo, conocimiento interdisciplinario/genérico sobre tecnologías y organizaciones, conocimiento sobre seguridad informática y protección de datos, conocimientos especializados sobre actividades y procesos de fabricación, programación informática y habilidades de codificación, conocimientos especializados sobre tecnologías, conocimiento sobre ergonomía y comprensión de los asuntos jurídicos.

También Coskun et al. (2019) y Sackey et al. (2017) concluyen que las nuevas capacidades requeridas por los ingenieros industriales en la Industria 4.0 son en áreas como: big data y análisis de datos, nuevas interfaces hombre-máquina, tecnologías de transferencia digital a física, comunicación y redes de datos, sistemas de posicionamiento global, sistemas de radiofrecuencia (RFID, por sus siglas en inglés), sistemas de planeación de recursos empresariales (ERP), la consigna electrónica (e-consignee), cómputo en la nube, realidad aumentada y sistemas logísticos autónomos.

Desde el contexto de la manufactura inteligente, autores como Frank et al. (2019) y Zhong et al. (2017) identificaron que tecnologías como el internet de las cosas, cómputo en la nube, big data, análisis de datos, sistemas ciberfísicos y TIC se están utilizando para la manufactura inteligente.

Con respecto a las competencias genéricas que debe desarrollar un ingeniero industrial, este estudio sugiere que se consideren como principales competencias las que menciona el Foro Económico Mundial (2018a). La figura 1 muestra un resumen de las competencias que debe adquirir el ingeniero industrial en su formación profesional para enfrentar los desafíos de la Industria 4.0. 


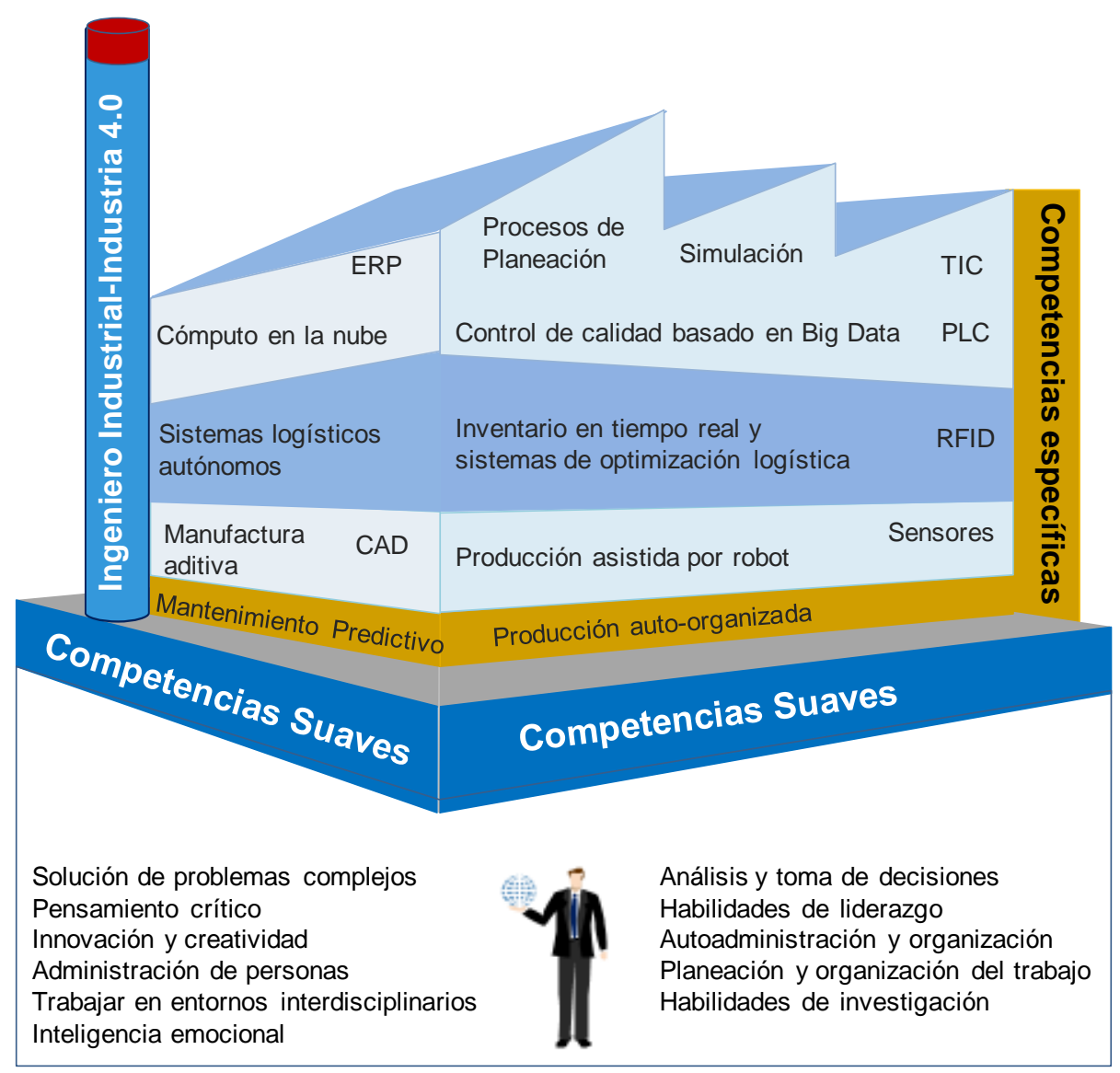

Fuente: Coskun et al. (2019); Foro Económico Mundial (2018a); Frank et al. (2019); Karre et al. (2017); Sackey y Bester (2016); Sackey et al. (2017); Zhong et al. (2017)

Figura 1. Competencias del ingeniero industrial

Bajo este panorama, Onar et al. (2018) mencionan que los contenidos necesarios en los planes de estudio de las ingenierías se pueden clasificar en cuatro grupos principales para enfrentar a la Industria 4.0. El primer grupo está compuesto por tecnologías de recolección, almacenamiento y procesamiento de datos. El segundo grupo se centra en operaciones automatizadas de valor agregado. El tercer grupo está compuesto de conocimiento de dominio (conocimiento técnico que incluye las aplicaciones y herramientas avanzadas en dominios específicos), y el grupo final es la innovación y el espíritu empresarial.

\section{Caso análisis curricular UAEH}

Sauza et al. (2017) mencionan que en las universidades de todo el mundo, en las áreas de ingeniería, se están desarrollando estrategias y acciones para incluir en sus planes de estudio los temas relacionados con la Industria 4.0. Para la presente investigación se consideró el análisis del programa de estudios en ingeniería industrial que oferta la Universidad Autónoma del Estado de Hidalgo (UAEH), la cual se encuentra dentro de las principales diez universidades en México y en el lugar 1001+ del International THE-World University Rankings (Times Higher Education, 2018).

El modelo educativo de la UAEH establece siete competencias genéricas (UAEH, 2015), y el programa educativo de la licenciatura en ingeniería industrial considera cuatro competencias específicas. Cabe mencionar que la información específica sobre el modelo educativo de la UAEH y del programa de estudios de ingeniería industrial fue solicitada en la Dirección de Educación Superior de esta institución. Las competencias genéricas y específicas para los estudiantes de ingeniería industrial se integran en tres 
niveles, donde el nivel tres implica el parámetro de mayor complejidad. En la tabla III se muestran las competencias que deben desarrollar los estudiantes de ingeniería industrial de la UAEH y la relación con las asignaturas que deben cursar.

Tabla III. Competencias genéricas y específicas del ingeniero industrial en la UAEH

\begin{tabular}{|c|c|c|c|c|}
\hline \multicolumn{2}{|c|}{ Competencias } & \multicolumn{3}{|c|}{ Nivel } \\
\hline Genéricas & Específicas & 1 & 2 & 3 \\
\hline Comunicación & $\begin{array}{l}\text { Administración de } \\
\text { la cadena de } \\
\text { suministro }\end{array}$ & $\begin{array}{l}\text { - Aprender a aprender } \\
\text { - Pre cálculo } \\
\text { - Química }\end{array}$ & $\begin{array}{l}\text { - Dibujo industrial } \\
\text { - Contabilidad } \\
\text { - Termo fluidos }\end{array}$ & $\begin{array}{l}\text { - Gestión de la } \\
\text { producción } \\
\text { - Administración de }\end{array}$ \\
\hline $\begin{array}{l}\text { Uso de la } \\
\text { tecnología }\end{array}$ & & $\begin{array}{l}\text { - Interacción materia y } \\
\text { energía } \\
\text { - México multicultural } \\
\text { - Lenqua extranjera }\end{array}$ & $\begin{array}{l}\text { - Máquinas eléctricas } \\
\text { - Desarrollo } \\
\text { sustentable } \\
\text { - Lenqua extranjera }\end{array}$ & $\begin{array}{l}\text { recursos humanos } \\
\text { - Distribución de planta } \\
\text { - Gestión de la calidad } \\
\text { - Ergonomía }\end{array}$ \\
\hline Creatividad & $\begin{array}{l}\text { Ubicación y } \\
\text { distribución de las } \\
\text { organizaciones }\end{array}$ & $\begin{array}{l}\text { - Estadística y } \\
\text { probabilidad } \\
\text { - Cálculo }\end{array}$ & $\begin{array}{l}\text { - Administración y } \\
\text { planeación estratégica } \\
\text { - Álaebra lineal }\end{array}$ & $\begin{array}{l}\text { - Logística y cadena de } \\
\text { suministro } \\
\text { - Optativa I }\end{array}$ \\
\hline Formación & $\begin{array}{l}\text { productivas de } \\
\text { bienes y servicios }\end{array}$ & $\begin{array}{l}\text { - Tecnología } \\
\text { informática } \\
\text { - Teoría de los }\end{array}$ & $\begin{array}{l}\text { - Procesos de } \\
\text { producción } \\
\text { - Mantenimiento }\end{array}$ & $\begin{array}{l}\text { - Evaluación de } \\
\text { proyectos } \\
\text { - Liderazgo }\end{array}$ \\
\hline $\begin{array}{l}\text { Pensamiento } \\
\text { crítico }\end{array}$ & $\begin{array}{l}\text { Diseño, } \\
\text { implementación y } \\
\text { control de las }\end{array}$ & $\begin{array}{l}\text { Sistemas } \\
\text { - Estudio de trabajo } \\
\text { - Estadística inferencial }\end{array}$ & $\begin{array}{l}\text { - Evaluación ambiental } \\
\text { - Seguridad e higiene } \\
\text { - Programación }\end{array}$ & $\begin{array}{l}\text { - Servicio social } \\
\text { - Optativa II } \\
\text { - Optativa III }\end{array}$ \\
\hline $\begin{array}{l}\text { Liderazgo } \\
\text { colaborativo }\end{array}$ & $\begin{array}{l}\text { condiciones de } \\
\text { trabajo óptimas }\end{array}$ & $\begin{array}{l}\text { - Ecuaciones } \\
\text { diferenciales } \\
\text { - Estudio de materiales } \\
\text { - Mecánica }\end{array}$ & $\begin{array}{l}\text { matemática y } \\
\text { simulación } \\
\text { - Análisis económico } \\
\text { - Calidad }\end{array}$ & $\begin{array}{l}\text { - Prácticas } \\
\text { profesionales }\end{array}$ \\
\hline Ciudadanía & $\begin{array}{l}\text { Diseño e } \\
\text { implementación y } \\
\text { control de los } \\
\text { sistemas de calidad }\end{array}$ & $\begin{array}{l}\text { - Metodología de la } \\
\text { Investigación }\end{array}$ & - Manufactura & \\
\hline
\end{tabular}

Fuente: UAEH-Ingeniería Industrial (2019); UAEH (2015)

\subsection{Competencias genéricas}

Comunicación. El objetivo es desarrollar en el estudiante la capacidad de comunicación que le permita establecer vínculos con su entorno social, cultural, político y económico.

Formación. Se busca que el ingeniero industrial integre los contenidos de aprendizaje en diversas situaciones para la solución de problemas a través del empleo de estrategias centradas en el aprendizaje.

Pensamiento crítico. El ingeniero aplica el pensamiento crítico y autocrítico para identificar, plantear y resolver problemas por medio de los procesos de abstracción, análisis y síntesis.

Creatividad. Esta competencia busca aplicar la creatividad para detectar, formular y solucionar problemas de forma original e innovadora, a través de la integración de contenidos y el uso de estrategias didácticas que generen el pensamiento divergente, investigativo, cooperativo e innovador.

Liderazgo colaborativo. El ingeniero industrial debe aplicar el liderazgo colaborativo para identificar y desarrollar ideas y proyectos del campo profesional y social por medio de los procesos de planeación estratégica y toma de decisiones, asegurando el trabajo en equipo, la motivación y la conducción hacia metas comunes.

Ciudadanía. El ingeniero debe conducirse ante los distintos colectivos de acuerdo con los principios generales de respeto a la diversidad cultural con responsabilidad social y compromiso ciudadano. 
Uso de la tecnología. Promueve aplicar las TIC como herramienta de apoyo para la solución de problemas del campo profesional y social a través del uso apropiado de recursos y metodologías para el desarrollo del aprendizaje, la comunicación, la formación disciplinar y la investigación.

\subsection{Competencias específicas}

Administración de la cadena de suministro. En esta competencia se busca que el ingeniero industrial evalúe la operatividad de las empresas productivas y de servicios para garantizar la producción óptima, disponibilidad y fiabilidad de sus equipos, la rentabilidad de nuevos productos o proyectos a través de técnicas de administración de la producción, del mantenimiento y de la evaluación de proyectos respetando las políticas de la empresa en materia de calidad, así como a las disposiciones legales nacionales e internacionales aplicables al medio ambiente y desarrollo sustentable.

En el contexto de la Industria 4.0 el enfoque de esta competencia es hacia la cadena de suministro inteligente, donde se incluyen los productos inteligentes, sensores, tecnología de RFID, entre otros. A partir de que la mayoría de los procesos en la cadena de suministro generan datos, el análisis big data se considera una herramienta primaria para alcanzar ventajas competitivas. En este sentido, el análisis big data es responsable de los avances realizados en diferentes componentes de la Industria 4.0, tales como la logística inteligente, fábricas inteligentes, el análisis de clientes, las ciudades inteligentes y los sistemas de transporte inteligentes (Lorenz et al., 2015; Queiroz y Telles, 2018). Estas habilidades en la cadena de suministro pertenecen principalmente a las competencias duras relacionadas con aspectos que van desde los procesos de planeación hasta el control de la calidad basado en big data.

Ubicación y distribución de las organizaciones productivas de bienes y servicios. Esta competencia busca desarrollar en el ingeniero industrial la capacidad de evaluar la ubicación y distribución de las organizaciones productivas de bienes y servicios, optimizando los costos de las mismas, a través de la aplicación de técnicas de localización y distribución de planta. En el entorno de la Industria 4.0 esta competencia se relaciona con la administración de sistemas ciber-físicos basados en habilidades como la simulación, ERP, los sistemas de optimización logística, CAD (Diseño asistido por ordenador), el cómputo en la nube y la producción auto-organizada.

Diseño, implementación y control de los sistemas de calidad. En esta competencia se incluye la implementación o mejora de sistemas de calidad en empresas productivas o de servicios, utilizando técnicas estadísticas, metodologías, herramientas y sistemas de administración de calidad, tomando en cuenta las normas nacionales e internacionales vigentes. Con relación a la Industria 4.0, Lorenz et al. (2015) mencionan que los sistemas de control de calidad se basarán en big data, ya que los algoritmos basados en datos históricos y datos en tiempo real identificarán problemas de calidad y reducirán fallas en los productos. Esta competencia, en general, incluye habilidades duras apoyadas en sensores, RFID, manufactura aditiva, control de calidad basado en big data, simulación y el mantenimiento predictivo.

Diseño, implementación y control de condiciones de trabajo óptimas. Esta competencia cubre el diseño de las condiciones óptimas en los entornos de trabajo, evaluando las áreas laborales en las organizaciones, mediante herramientas que involucran el estudio del trabajo, la ergonomía, seguridad e higiene conforme a los estándares nacionales e internacionales aplicables. Bajo el contexto de la Industria 4.0, Bortolini et al. (2020) mencionan que la representación virtual (simulación) puede ser de gran ayuda para analizar y mejorar los procesos de fabricación y ensamblaje, así como diseñar las condiciones óptimas de los entornos de trabajo.

En la tabla IV se resumen las competencias que debe desarrollar un estudiante de la licenciatura en ingeniería industrial de acuerdo al programa de estudios de la UAEH, así como las competencias que se deben considerar para enfrentar los desafíos de la Industria 4.0 con el objetivo de que el ingeniero industrial pueda insertarse en la manufactura inteligente. 
Tabla IV. Enlace de competencias

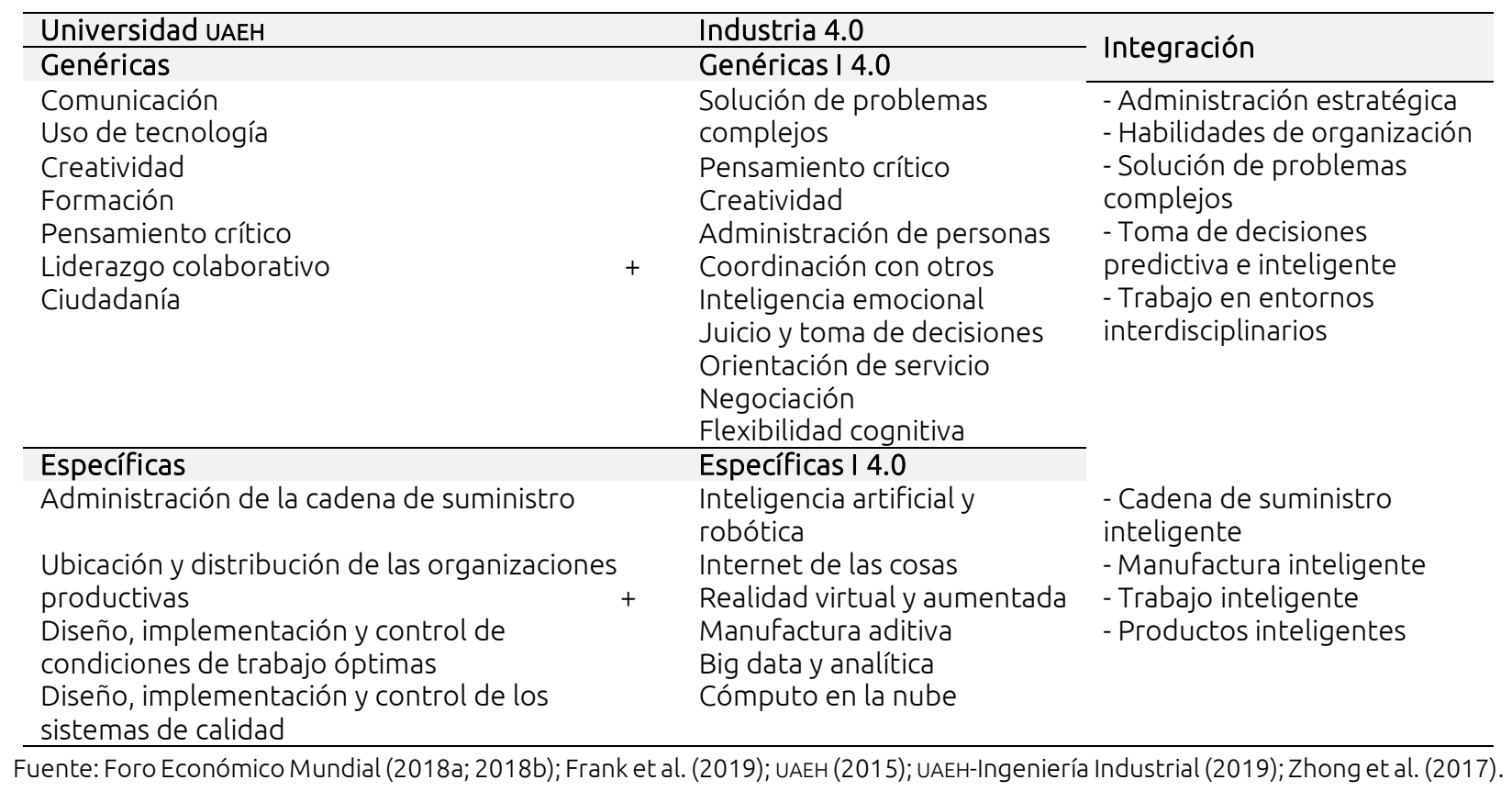

\section{Discusión y conclusiones}

Como se mencionó en apartados anteriores, los fundamentos de la Industria 4.0 son las tecnologías emergentes que tienen como fin desarrollar sistemas de manufactura inteligente. En este sentido, con base en los hallazgos de este estudio se sugiere que la formación del ingeniero industrial debe estar centrada en tecnologías (competencias específicas) como: big data, análisis de datos, sistema de ejecución de manufactura, producción inalámbrica, planificación de recursos empresariales, comunicación máquina a máquina, realidad virtual, simulación de procesos, inteligencia artificial para el mantenimiento predictivo y para la planeación de la producción, robots industriales, vehículos guiados autónomos, identificación automática de no conformidades en producción, identificación y trazabilidad de materias primas, identificación y trazabilidad de productos finales, manufactura aditiva, líneas flexibles y autónomas, y tecnología orientada al servicio.

Para desarrollar estas competencias varios esfuerzos han sido detonados, en particular en la educación superior, tratando de comprender mejor la Industria 4.0. Tales esfuerzos en la educación superior han comenzado con iniciativas llamadas Fábrica de Aprendizaje (Learning Factory) -que corresponden a unidades de producción y montaje pequeñas y flexibles para la práctica y la capacitación (Mourtzis et al., 2018).

Otra iniciativa es Teaching Factory, que ha emergido como un paradigma prometedor para integrar los entornos de aprendizaje y trabajo. El cual es un espacio de aprendizaje no anclado geográficamente que interconecta de forma remota equipos de ingeniería y estudiantes que trabajan juntos en proyectos de la vida real (Mavrikios et al., 2019).

Desde la perspectiva de los estudiantes en ingeniería industrial en México, la Industria 4.0 representa una ventana de oportunidad con un potencial considerable para los estudiantes, ya que este paradigma permitiría la convergencia de dos sectores estratégicos claramente competitivos del país: manufactura y TIC. Estos sectores estratégicos han permitido que México sea conocido como un centro de manufactura de clase mundial, que exporta más de mil millones de dólares por día y el $50 \%$ de estas exportaciones son productos manufacturados, de los cuales gran parte son tecnologías altamente sofisticadas. 
Además, los acuerdos comerciales internacionales, junto con la correlación entre el peso mexicano y el dólar estadounidense, más la experiencia obtenida de las industrias de electrónica y automotriz, han creado una zona altamente competitiva para el desarrollo de una industria de exportación de manufacturas.

A la luz de esta cuarta revolución industrial es importante comprender la posición geoestratégica que tiene México y aprovechar su potencial en beneficio de las empresas mexicanas y de los futuros ingenieros. De tal forma que una parte importante de las tareas de las universidades mexicanas es adaptar la educación superior a los requisitos de la Industria 4.0, en particular la educación en ingeniería, con el objetivo de desarrollar las competencias y habilidades necesarias en los estudiantes para que asimilen las nuevas tecnologías y las mejores prácticas internacionales destinadas a crear un alto valor agregado para México.

Bajo este panorama, la Secretaría de Economía estableció un conjunto de estrategias en su mapa de ruta para la Industria 4.0, llamada Crafting the future: a roadmap for Industry 4.0 in Mexico, que tiene como fin ayudar a la industria mexicana, a las diferentes cadenas de suministro de los sectores estratégicos, a la academia, a grupos de investigación y al gobierno para que prioricen las tendencias y tecnologías necesarias que soportan la Industria 4.0. Estas estrategias están centradas en educación, tecnología, economía digital y proyectos (Secretaría de Economía de México, 2016).

Finalmente, el propósito de esta investigación es proporcionar un panorama general sobre las competencias genéricas y específicas que debe desarrollar un ingeniero industrial con el fin de coadyuvar con las universidades mexicanas en el diseño y la preparación de los contenidos de estudio que converjan con los requisitos que demandan los sectores estratégicos de manufactura en México y la Industria 4.0. En primer lugar encontramos que el Foro Económico Mundial enlista diez competencias genéricas para permanecer en la cuarta revolución industrial. Para el caso de las competencias específicas (competencias en tecnologías emergentes) que debe desarrollar un ingeniero industrial detectamos que éstas deben estar enfocadas en los sistemas de manufactura inteligente, cadenas de suministro inteligente, trabajo inteligente y productos inteligentes.

Futuras investigaciones sobre este tema se pueden centrar en cómo se están desarrollando e implementando las iniciativas o estrategias de Learning Factory y Teaching Factory para el desarrollo de competencias y cómo se han beneficiado los estudiantes en su integración a la Industria 4.0.

\section{Referencias}

Acosta, E. P. (2016). Aproximación a las cualificaciones profesionales en la Industria 4.0. Fundación 1 de Mayo, (22).

Bortolini, M., Faccio, M., Gamberi, M. y Pilati, F. (2020). Motion Analysis System (MAs) for production and ergonomics assessment in the manufacturing processes. Computers \& Industrial Engineering, 139. https://doi.org/10.1016/i.cie.2018.10.046

Chong, S., Pan, G.-T., Chin, J., Show, P. L., Yang, T. C. K. y Huang, C.-M. (2018). Integration of 3D printing and industry 4.0 into engineering teaching. Sustainability, 10(11), 1-13. https://doi.org/10.3390/su10113960

Coskun, S., Kayıkcl, Y. y Gencay, E. (2019). Adapting engineering education to Industry 4.0 Vision. Technologies, 7(1), 1-10. https://doi.org/10.3390/technologies7010010

Dalenogare, L. S., Benitez, G. B., Ayala, N. F. y Frank, A. G. (2018). The expected contribution of Industry 4.0 technologies for industrial performance. International Journal of Production Economics, 204, 383-394. https://doi.org/10.1016/i.ijpe.2018.08.019

Foro Económico Mundial. (2018a). 10 skills you'll need to survive the rise of automation. https://www.weforum.org/agenda/2018/07/the-skills-needed-to-survive-the-robot-invasion-of-theworkplace 
Foro Económico Mundial. (2018b). Readiness for the Future of Production Report 2018.

https://www.weforum.org/reports/readiness-for-the-future-of-production-report-2018

Foro Económico Mundial. (2019). Fourth Industrial Revolution.

https://intelligence.weforum.org/topics/a1Gb0000001RIhBEAW?tab=publications

Frank, A. G., Dalenogare, L. S. y Ayala, N. F. (2019). Industry 4.0 technologies: Implementation patterns in manufacturing companies. International Journal of Production Economics, 210, $15-26$.

https://doi.org/10.1016/i.ijpe.2019.01.004

Ghobakhloo, M. (2018). The future of manufacturing industry: a strategic roadmap toward Industry 4.0. Journal of Manufacturing Technology Management, 29(6), 910-936. https://doi.org/10.1108/JMTM-02-2018$\underline{0057}$

Gronau, N., Ullrich, A. y Teichmann, M. (2017). Development of the Industrial IoT Competences in the Areas of Organization, Process, and Interaction based on the Learning Factory Concept. Procedia Manufacturing, 9, 264-261. https://doi.org/10.1016/i.promfg.2017.04.029

Grzybowska, K. y Lupicka, A. (2018). Key competencies for Industry 4.0. Economics \& Management Innovations, 1(1), 250-253.

Jerman, A., Pejić Bach, M. y Bertoncelj, A. (2018). A bibliometric and topic analysis on future competences at smart factories. Machines, 6(3), 41. https://doi.org/10.3390/machines6030041

Karre, H., Hammer, M., Kleindienst, M. y Ramsauer, C. (2017). Transition towards an Industry 4.0 state of the LeanLab at Graz University of Technology. Procedia Manufacturing, 9, 206-213.

https://doi.org/10.1016/i.promfg.2017.04.006

Liboni, L. B., Cezarino, L. O., Jabbour, C. J. C., Oliveira, B. G. y Stefanelli, N. O. (2019). Smart industry and the pathways to HRM 4.0: implications for SCM. Supply Chain Management: An International Journal, 24(1), 124146. https://doi.org/10.1108/SCM-03-2018-0150

Lorenz, M., Rüßmann, M., Strack, R., Lueth, K. y Bolle, M. (2015). Man and Machine in Industry 4.0. https://www.bcg.com/industries/engineered-products-infrastructure/man-machine-industry-4.0.aspx

Mavrikios, D., Georgoulias, K. y Chryssolouris, G. (2019). The teaching factory network: a new collaborative paradigm for manufacturing education. Procedia Manufacturing, 31, 398-403.

https://doi.org/10.1016/i.promfg.2019.03.062

Mesquita, D., Lima, R. M., Flores, M. A., Marinho-Araujo, C. y Rabelo, M. (2015). Industrial engineering and management curriculum profile: developing a framework of competences. International Journal of Industrial Engineering and Management, 6(3), 121-131.

Motyl, B., Baronio, G., Uberti, S., Speranza, D. y Filippi, S. (2017). How will change the future engineers' skills in the industry 4.0 framework? A questionnaire survey. Procedia Manufacturing, 11, 1501-1509.

https://doi.org/10.1016/i.promfg.2017.07.282

Mourtzis, D., Vlachou, E., Dimitrakopoulos, G. y Zogopoulos, V. (2018). Cyber-physical systems and education 4.0 -the teaching factory 4.0 concept. Procedia Manufacturing, 23, 129-134.

https://doi.org/10.1016/j.promfg.2018.04.005

Mourtzis, D. (2018). Development of skills and competences in manufacturing towards education 4.0: a teaching factory approach. En J. Ni, V. D. Majstorovic y D. Djurdjanovic (Eds.), AMP 2018, Actas de 3rd International Conference on the Industry 4.0 Model for Advanced Manufacturing (pp. 194-210). Springer. https://doi.org/10.1007/978-3-319-89563-5 15 
Onar, S. C., Ustundag, A., Kadaifci, C. K. y Oztaysi, B. (2018). The changing role of engineering education in industry 4.0 era. En A. Ustundag y E. Cevikcan (Eds.), Industry 4.0: managing the digital transformation (pp. 137-151). Springer. https://doi.org/10.1007/978-3-319-57870-5 8

Prifti, L., Knigge, M., Kienegger, H. y Krcmar, H. (2017). A competency model for "Industrie 4.0 " employees. En J. M. Leimeister y W. Brenner (Eds.), Actas 13th International Conference on Wirtschaftsinformatikk pp. 46-60. Gallen, Switzerland.

Queiroz, M. M. y Telles, R. (2018). Big data analytics in supply chain and logistics: an empirical approach Maciel. The International Journal of Logistics Management, 29(2), 767-783. https://doi.org/10.1108/IJLM-05$\underline{2017-0116}$

Ramingwong, S. y Manopiniwes, W. (2019). Supportment for organization and management competences of ASEAN community and European Union toward Industry 4.0. International Journal of Advanced and Applied Sciences, 6(2), 81-86. https://doi.org/10.21833/ijaas.2019.03.014

Sackey, S. M. y Bester, A. (2016). Industrial engineering curriculum in Industry 4.0 in a South African context. South African Journal of Industrial Engineering, 27(4), 101-114. https://doi.org/10.7166/27-4-1579

Sackey, S., Bester, A. y Adams, D. (2017). Industry 4.0 learning factory didactic design parameters for industrial engineering education in South Africa. South African Journal of Industrial Engineering, 28(1), 114124. https://doi.org/10.7166/28-1-1584

Sánchez, F., Soler, A., Martín, C., López, D., Ageno, A., Cabré, J., García, J., Aranda, J. y Gibert, K. (2018). Competency maps: an effective model to integrate professional competencies across a STEM curriculum. Journal of Science Education and Technology, 27(5), 448-468. https://doi.org/10.1007/s10956-018-9735-3

Saucedo-Martínez, J. A., Pérez-Lara, M., Marmolejo-Saucedo, J. A., Salais-Fierro, T. E. y Vasant, P. (2017). Industry 4.0 framework for management and operations: a review. Journal of Ambient Intelligence and Humanized Computing, 9(3), 789-801. https://doi.org/10.1007/s12652-017-0533-1

Sauza, J. B., D’Antonio, G. y Chiaberta, P. (2017). A novel approach for teaching it tools within learning factories. Procedia Manufacturing, 9, 175-181. https://doi.org/10.1016/j.promfg.2017.04.049

Secretaría de Economía de México. (2016). Crafting the future: a roadmap for Industry 4.0 in Mexico. Autor.

Terrés, J. I., Viles, E., Lleó, Á. y Santos, J. (2017). Competencias Profesionales 4.0. Tecnum Universidad de Navarra.

Times Higher Education. (2018). World University Rankings. https://www.timeshighereducation.com/worlduniversity-rankings

Tuning-América Latina. (2007). Reflexiones y perspectivas de la Educación Superior en América Latina. P. Beneitone, C. Esquetini, J. González, M. M. Maletá, G. Siufi y R. Wagenaar (Eds.). Publicaciones de la Universidad de Deusto.

Universidad Autónoma del Estado de Hidalgo-Ingeniería Industrial. (2019). Programa Educativo de la Licenciatura en Ingeniería Industrial.

https://www.uaeh.edu.mx/campus/icbi/oferta/licenciaturas/ing industrial.html

Universidad Autónoma del Estado de Hidalgo. (2015). Modelo Educativo UAEH.

https://www.uaeh.edu.mx/modelo educativo/

Zhong, R. Y., Xu, X., Klotz, E. y Newman, S. T. (2017). Intelligent Manufacturing in the Context of Industry 4.0: A Review. Engineering, 3(5), 616-630. https://doi.org/10.1016/J.ENG.2017.05.015 
Zlatkin-Troitschanskaia, O., Pant, H. A., Lautenbach, C., Molerov, D., Toepper, M. y Brückner, S. (2017). Modeling and measuring competencies in higher education: approaches to challenges in higher education policy and practice. Springer. https://doi.org/10.1007/978-3-658-15486-8 